diagnosis of peroxisomal $\beta$-oxidation defects. J Clin Invest 97:681-688

Ravnik-Glavac M, Glavac D, Dean M (1994) Sensitivity of single-stranded conformation polymorphism and heteroduplex method for mutation detection in the cystic fibrosis gene. Hum Mol Genet 3:801-807

Sanger F, Nicklen S, Coulson RA (1977) DNA sequencing with chain terminating inhibitors. Proc Natl Acad Sci USA 74:5463-5467

Shani N, Watkins PA, Valle D (1995) PXA1, a possible Saccharomyces cerevisiae ortholog of the human adrenoleukodystrophy gene. Proc Natl Acad Sci USA 92:6012-6016

Sheffield VC, Beck JS, Kwitek AE, Sandstrom DW, Stone EM (1993) The sensitivity of single-strand conformation polymorphism analysis for the detection of single base substitutions. Genomics 16:325-332

Shimozawa N, Suzuki Y, Orii T, Moser A, Moser HW, Wanders RJA (1993) Standardization of complementation grouping of peroxisome-deficient disorders and the second patient with peroxisomal assembly factor-1 (PAF-1) defect. Am J Hum Genet 52:843-844

Shimozawa N, Suzuki Y, Tomatsu S, Tsukamoto T, Osumi T, Fujiki Y, Kamijo K, et al (1996) Correction by gene expression of biochemical abnormalities in fibroblasts from Zellweger patients. Pediatr Res 39:812-815

Tan X, Titorenko VI, van der Klei IJ, Sulter GJ, Haima P, Waterham HR, Evers M, et al (1995) Characterization of peroxisome-deficient mutants of Hansenula polymorpha. Curr Genet 28:248-257

Yajima S, Suzuki Y, Shimozawa N, Yamaguchi S, Orii T, Fujiki Y, Osumi T, et al (1992) Complementation study of peroxisome-deficient disorders by immunofluorescence staining and characterization of fused cells. Hum Genet 88: 491-499

Address for correspondence and reprints: Dr. Barbara Paton, Department of Chemical Pathology, Women's and Children's Hospital, 72 King William Road, North Adelaide SA 5006, Australia. E-mail: patonb@wch.sa.gov.au

*Present affiliation: Molecular Pathology Unit, Institute of Medical and Veterinary Science, Adelaide.

* * Present affiliation: School of Life Sciences, Queensland University of Technology, Brisbane.

(1) 1997 by The American Society of Human Genetics. All rights reserved. 0002-9297/97/6006-0033\$02.00

Am. J. Hum. Genet. 60:1539-1542, 1997

\section{Disease Relevance of the So-Called Secondary Leber Hereditary Optic Neuropathy Mutations}

To the Editor:

In the August issue of the Journal, Mackey et al. (1996) addressed the open question of the pathogenic role of the mtDNA mutation at nucleotide position (np) 15257 in Leber hereditary optic neuropathy (LHON). This was done by investigating a total of 159 LHON families from Australia/New Zealand and from several European countries. As a major result, the 15257 mutation was found in $6(4 \%)$ of 159 families, and in every one of these instances it was associated with one of the established primary LHON mutations, at np 11778 (in 4 [5\%] of 78 families), at np 3460 (in 1 [7\%] of 14 families), and at np 14484 (in 1 [4\%] of 23 families). Because the 15257 mutation did not occur in isolation of an established primary LHON mutation, Mackey et al. questioned whether there is a primary pathogenic role for this mutation in the cytochrome $b$ gene. However, they also discussed possible doubts about this conclusion. As one of the points, they cited a report by our group (Obermaier-Kusser et al. 1994) on a German LHON family that carries the 15257 mutation but lacks any of the three established primary mutations. We would like to comment on this point. In the cited paper, we described 17 families and sporadic cases in which the mutation pattern included at least one primary or intermediate mutation. The 15257 mutation was found in three index patients; in two cases in association with the 11778 or 14484 mutation and in one case without any of the three primary mutations. Maternal inheritance and male predominance in combination with the clinical features confirm the classification of LHON in this latter family. Of the eight families tested in that paper, the singleton 15257 family showed the highest penetrance (56\%, all males). Meanwhile, we identified 38 further LHON index cases. Our total LHON collective $(n=55)$ now consists of 24 carriers of 11778,5 carriers of 3460 , and 21 carriers of 14484 ; the 15257 mutation is found three times associated with np 11778, six times associated with np 14484, and five times without any of the established primary mutations. The percentage of LHON patients carrying the 15257 mutation $(25 \%)$ by far exceeds the finding by Mackey et al. (1996). In the five cases without primary mutation, the 15257 mutation behaves like a primary LHON mutation; however, we cannot exclude the possibility that another, still-unknown primary mutation is present. Two other groups also reported singleton $15257 \mathrm{LHON}$ cases (Howell et al. 1993; Brown et al. 1995), and, in these studies, other pathogenic mtDNA mutations could be excluded by screening most of the mtDNA coding regions.

The pathogenicity of the 15257 mutation is also discussed with respect to its haplogroup specificity. This mutation is nearly always associated with the mutations at nps 4216 and 13708 (Johns and Berman 1991; Brown et al. 1992). Mackey et al. (1996, p. 484) concluded that "the increased frequency of the 15257 mutation among LHON patients can be explained solely in terms of population history and genetics, rather than as a reflection of its pathogenic role." In order to look for an association of certain haplogroups with distinct neurodegenerative disorders, we currently are investigating 
the entire mitochondrial genome of our total LHON collective $(n=55)$ and of eight DIDMOAD (diabetes insipidus, diabetes mellitus, optic atrophy, and deafness) patients by use of SSCP analysis and direct sequencing of PCR-amplified fragments. The sequence data sets (including variants of all ND, tRNA, and cyt $b$ genes) are analyzed with regard to common mutation clusters (haplogroups) in the respective disease groups. The haplogroup frequencies in disease groups are compared with those in a group of healthy German blood donors $(n=67)$. Of the 67 control subjects, none carried the 15257 mutation. Of the 14 LHON cases who were 15257 positive, $12(86 \%)$ were found in association with the mutations at nps 4216 and 13708 . These mutations are commonly in association with further ND gene polymorphisms at nps 10398,11251 , and 12612 (fig. 1), forming a distinct haplogroup (termed " $A$ " in fig. 1) that, because of a DdeI restriction-site gain at np 10394 and a $B s t$ NI restriction site loss at np 13704 , presumably is consistent with the European haplogroup J (Torroni et al. 1994). It represents $6 \%$ (4 of 67 controls) of our healthy German control group, a frequency slightly lower than those described for other Caucasian populations (Torroni et al. 1994, 1996b). In contrast to the

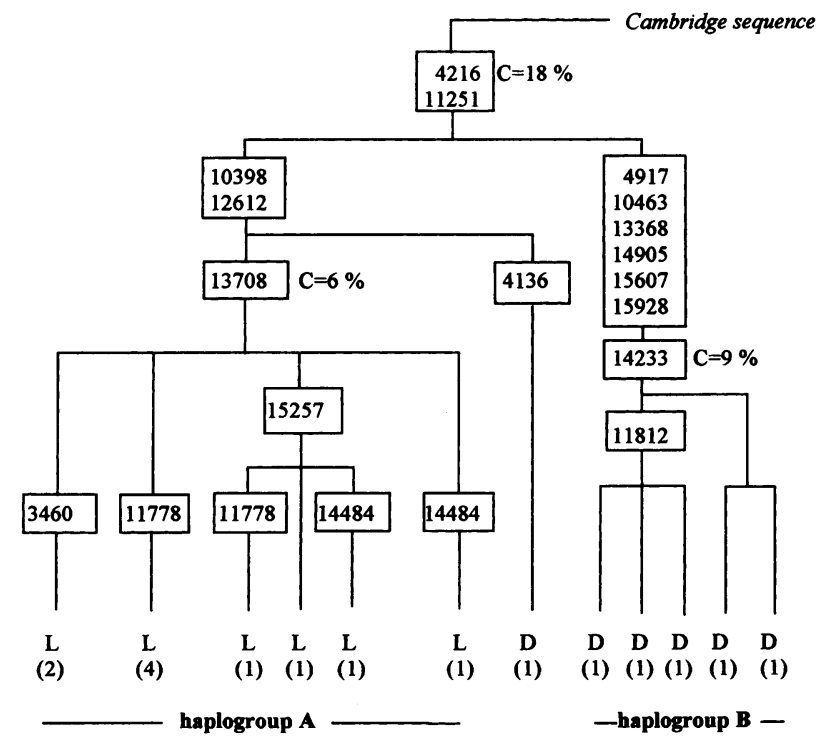

Figure 1 mtDNA haplotypes of 4216-positive LHON and DIDMOAD patients. Sequence data included in this cladogram come from the mtDNA screening of 17 LHON (L) patients, 8 DIDMOAD (D) patients, and 67 controls (C); mtDNA haplotype analysis of the remaining LHON cases $(n=38)$ is currently under investigation. 4216-wild-type patients (i.e., seven LHON patients and two DIDMOAD patients) are not included in this figure. Number of LHON or DIDMOAD patients belonging to the distinct branches is indicated in parentheses. All subbranches associated with DIDMOAD patients were defined by further coding-region variants not depicted here. The different levels of mutation clusters are not correlated with specific genetic distances. primary LHON mutations at np 3460,11778 , or 14484 , which are also found in association with other haplogroups, the 15257 mutation is associated nearly exclusively with haplogroup A, thus creating a highly pathogenic subbranch of this haplogroup. However, two 15257-positive cases were found in association with $\mathrm{np}$ 4917. One of these patients did not harbor any of the common primary LHON mutations, a finding further arguing in favor of a pathogenic role of the 15257 mutation, even in the absence of the $4216+13708$ haplogroup background.

From a clinical point of view, Mackey et al. (1996, p. 482) discussed the possibility that LHON is not a single entity but can, in simplest terms, be subdivided into two broad groups. "In the first group the optic neuropathy is the most prominent, if not the exclusive, clinical deficit, although there may be extra-ophthalmologic abnormalities, including peripheral neuropathy, heart conduction defects, and a multiple sclerosis-like condition, in a small proportion of family members. In contrast, there are other families that present severe neurological abnormalities, which also are maternally inherited and which are more prominent than the optic neuropathy." Mackey et al. stated that these latter "LHONplus" families involve pathogenic mutations different from those that produce the more ophthalmologically limited disorder and that these mutations seem to create specific LHON-plus disorders. In this respect a recent publication by Rödel et al. (1996) is of interest. These authors investigated the association of the 13708 and 15257 mutations with neurodegenerative diseases distinct from LHON and without primary LHON mutations. Two hundred unselected neurology patients and 100 subjects suffering from multiple sclerosis (MS) were screened for both mutations. Seven neurology patients $(3.5 \%)$ and two patients with MS $(2 \%)$ were found to carry both the 13708 mutation and the 15257 mutation, indicating that these patients also belong, in all probability, to haplogroup A. Thus, the 15257 mutation seems to be frequently associated with neurological symptoms, confirming the pathogenic role of this side branch of haplogroup A, but cannot be regarded as exclusively an LHON mutation.

The same holds true for the so-called secondary LHON mutations at nps 4216, 4917, and 13708, which define distinct phylogenetic clusters also found in the healthy Caucasian population. As shown in figure 1 , subjects carrying the $4216+11251$ combination split into haplogroups $\mathrm{A}$ and $\mathrm{B}$; the latter is defined by variants at nps 4216, 11251, 4917, 10463, $13368,14905,15607,15928$, and 14233 and presumably is consistent with the European-specific haplogroup T (Torroni et al. 1996b). The established primary LHON mutations at nps 11778, 3460, and 14484 are not randomly distributed over the various 
haplogroups but accumulate in haplogroup A (fig. 1). Concerning our total LHON collective $(n=55), 14$ $(66 \%)$ of 21 cases who were 14484 positive, 11 (46\%) of 24 cases who were 11778 positive, and $2(40 \%)$ of 5 cases who were 3460 positive were found in association with a $4216+13708$ background. Explanations for this observation are under discussion (Brown et al. 1995; Howell et al. 1995; Torroni et al. 1996a). Howell et al. (1995) proposed two possible situations to explain the high frequency of LHON in haplogroup A. One is that the mtDNA variants defining haplogroup A as well as the respective primary LHON mutations had arisen multiple times during human evolution. Such a situation is not supported by the data available from the literature (Howell et al. 1995). The other extreme is the "single-origin model" of the secondary LHON mutations. If it is assumed that the 13708 mutation (vicariously representing haplogroup A) arose once within the population and, for example, that "the 11778 mutation arose late in the history of a non-13708 line of descent but arose early in that of the 13708 line, then the most likely sampling outcome will be a high proportion of $11778+13708$ LHON pedigrees" (Howell et al. 1995, p. 296). If this assumption is true, then it must be considered valid also for the other two primary LHON mutations, since not only the 11778 mutation but also the two other primary mutations, at nps 3460 and 14484, are concentrated in haplogroup A. Moreover, with respect to our LHON families, at least the 11778 mutation arose twice or more within the $4216+13708$ haplogroup, since sequence analysis of the D-loop region of four of our LHON cases shows that at least two types of 11778 carriers can be distinguished by different clusters of D-loop variants (Hofmann et al. 1997). Thus, it seems unlikely that the accumulation of primary LHON mutations in haplogroup A is due solely to population history of primary and secondary LHON mutations. On the other hand, we have no reasonable explanation for the frequent occurrence of primary LHON mutations within haplogroup A. Either this haplogroup tends to acquire additional mutations or represents per se a "locus minoris resistentiae" - that is, a locus of impaired oxidative phosphorylation capacity - thus favoring the manifestation of disease. The assumption that a defined mtDNA haplogroup represents a susceptibility factor for a given neurodegenerative disorder is confirmed by our ongoing study on patients suffering from DIDMOAD syndrome. DIDMOAD, in contrast to LHON, never has been shown to be maternally transmitted and is, therefore, unlikely to be caused by a primary mtDNA mutation of high penetrance. A DIDMOAD disease locus has been tentatively mapped to chromosome $4 \mathrm{p}$ (Polymeropoulos et al. 1994), although there is evidence for genetic heterogeneity (Collier et al. 1996). Nevertheless, we observe that six $(75 \%)$ of eight DIDMOAD patients carry the $4216+11251$ variants (fig. 1); one 4216 carrier belongs to a separated side branch of haplogroup A, and five 4216 carriers (or $63 \%$ of all investigated DIDMOAD patients) belong to haplogroup B (Hofmann et al. 1997); in contrast, the frequency in our German control group is only $9 \%$ (6 of 67 controls). Thus, the possibility might exist that severe mutations of both nuclear or mitochondrial origin are indeed randomly associated with all mitochondrial haplogroups but will reach disease quality - that is, manifestation or penetrance-mainly when associated with a certain mitochondrial haplogroup. If so, such a condition could have important implications for polygenic disorders such as diabetes, hypertension, etc.

We propose that, in contrast to the primary LHON mutations, which are obviously LHON specific, the so-called secondary LHON mutations are not pathognomonic for LHON but seem to create a susceptibility basis for certain neurodegenerative disorders in a way that, so far, is unknown. The term secondary LHON mutations, therefore, should be dropped, at least with respect to nps 4216,4917 , and 13708 .

\section{Sabine Hofmann, ${ }^{1,2}$ Reimar Bezold ${ }^{2}$ Michaela JaKSCH, ${ }^{1}$ Petra KaufHOld,${ }^{2}$ Bert Obermaier- KUSSER, ${ }^{1}$ and KLAUS-DiETER GERBITZ ${ }^{1,2}$ Institutes of ${ }^{1}$ Clinical Chemistry and ${ }^{2}$ Diabetes Research, Academic Hospital Schwabing, Munich}

\section{References}

Brown MD, Torroni A, Reckord CL, Wallace DC (1995) Phylogenetic analysis of Leber's hereditary optic neuropathy mitochondrial DNA's indicates multiple independent occurrences of the common mutations. Hum Mutat 6:311-325

Brown MD, Voljavec AS, Lott MT, Torroni A, Yang CC, Wallace DC (1992) Mitochondrial DNA complex I and III mutations associated with Leber's hereditary optic neuropathy. Genetics 130:163-173

Collier DA, Barrett TG, Curtis D, Macleod A, Arranz MJ, Maassen JA, Bundey S (1996) Linkage of Wolfram syndrome to chromosome 4p16.1 and evidence for heterogeneity. Am J Hum Genet 59:855-863

Hofmann S, Bezold R, Jaksch M, Obermaier-Kusser B, Mertens S, Kaufhold P, Rabl W, et al (1997) Wolfram (DIDMOAD) syndrome and Leber's hereditary optic neuropathy (LHON) are associated with distinct mitochondrial DNA haplotypes. Genomics 39:8-18

Howell N, Kubacka I, Halvorson S, Howell B, McCullough DA, Mackey D (1995) Phylogenetic analysis of the mitochondrial genomes from Leber hereditary optic neuropathy pedigrees. Genetics 140:285-302 
Howell N, Kubacka I, Halvorson S, Mackey D (1993) Leber's hereditary optic neuropathy: the etiological role of a mutation in the mitochondrial cytochrome b gene. Genetics 133: 133-136

Johns DR, Berman J (1991) Alternative, simultaneous complex I mitochondrial DNA mutations in Leber's hereditary optic neuropathy. Biochem Biophys Res Commun 174:13241330

Mackey DA, Oostra R-J, Rosenberg T, Nikoskelainen E, Bronte-Stewart J, Poulton J, Harding AE, et al (1996) Primary pathogenic mtDNA mutations in multigeneration pedigrees with Leber hereditary optic neuropathy. Am J Hum Genet 59:481-485

Obermaier-Kusser B, Lorenz B, Schubring S, Paprotta A, Zerres K, Meitinger T, Meire F, et al (1994) Features of mtDNA mutation patterns in European pedigrees and sporadic cases with Leber hereditary optic neuropathy. Am J Hum Genet 55:1063-1066

Polymeropoulos MH, Swift RG, Swift M (1994) Linkage of the gene for Wolfram syndrome to markers on the short arm of chromosome 4. Nat Genet 8:95-97

Rödel G, Laubhan R, Scheuerle A, Skowronek P, Haferkamp O (1996) Association of the LHON 13708 and 15257 mitochondrial DNA mutations with neurodegenerative diseases distinct from LHON. Eur J Med Res 1:491-494

Torroni A, Carelli V, Petrozzi M, Terracina M, Barboni P, Malpassi P, Wallace DC, et al (1996a) Detection of the mtDNA 14484 mutation on an African-specific haplotype: implications about its role in causing Leber hereditary optic neuropathy. Am J Hum Genet 59:248-252

Torroni A, Huoponen K, Francalacci P, Petrozzi M, Morelli L, Scozzari R, Obinu D, et al (1996b) Classification of European mtDNAs from an analysis of three European populations. Genetics 144:1835-1850

Torroni A, Lott MT, Cabell MF, Chen Y-S, Lavergne L, Wallace DC (1994) mtDNA and the origin of Caucasians: identification of ancient Caucasian-specific haplogroups, one of which is prone to a recurrent somatic duplication in the Dloop region. Am J Hum Genet 55:760-776

Address for correspondence and reprints: Dr. Sabine Hofmann, Institute für Klinische Chemie und Diabetesforschung, Krankenhaus Munchen-Schwabing, Kölner Platz 1, 80804 München, Germany. E-mail: diabetes@lrz.unimuenchen.de

(C) 01997 by The American Society of Human Genetics. All rights reserved. 0002-9297/97/6006-0034\$02.00

Am. J. Hum. Genet. 60:1542-1544, 1997

\section{A Mutation in the MTM1 Gene Invalidates a Previous Suggestion of Nonallelic Heterogeneity in X-Linked Myotubular Myopathy}

To the Editor:

We reported previously in the Journal (Samson et al. 1995, p. 120) the study of a family with a single case of myotubular myopathy, in which linkage analysis, combined with examination of muscle biopsies in $\mathrm{fe}$ males for determination of carrier status, led us to "strongly suggest genetic heterogeneity" of this Xlinked disease, which previously had been mapped to Xq28 (MTM1 locus; OMIM 310400; Dahl et al. 1995; for a review, see Wallgren-Pettersson et al. 1995). Very recently, we have identified an Xq28 gene, coding for a putative tyrosine phosphatase, that was found mutated in many patients with X-linked myotubular myopathy (J. Laporte, unpublished data). We decided to reanalyze family $Z$ for the markers closest to this gene and to search for a mutation in the proband, using SSCP analysis on the exons that have been characterized to date (Laporte et al. 1996). We report here that a missense mutation indeed was found in the proband but was not present in his mother and in three other females thought to be carriers (see fig. 1), on the basis of detection of some small fibers with centrally located nuclei in the muscle biopsies of these individuals.

In our previous analysis, we reported that the unaffected boy (III.6) had inherited the same maternal alleles at the FRAXAC2 and DXS305 loci flanking MTM1 as were seen in the proband (III.4) and his putative carrier sister (III.3). However, a double recombination within FRAXAC2-DXS305, an interval of $\sim 10 \mathrm{cM}$, could not be formally excluded (Samson et al. 1995). The study of three microsatellite markers much closer to the MTM1 gene (Hu et al. 1996a; Laporte et al. 1996) now confirms that the same haplotype indeed was transmitted to all four children of II. 4 and also is present in the putative carriers (II.1 and II.2).

SSCP analysis was performed on five exons covering $752 \mathrm{bp}$ of coding sequence. For the exon encoding amino acids 370-438 (exon c, according to Laporte et al. 1996), we found four different band patterns (fig. 2). Sequencing of variant bands revealed the presence of two base-pair changes in the $5^{\prime}$ end of the following intron, defining three alleles (fig. 3). Alleles 1 and 2 differ at the consensus donor site and are common variants in the normal population (J. Laporte, unpublished data). Allele 3 , with an additional intronic change, is much rarer. It is present in the grandmother and also in III.2, in the latter of whom it must derive from the married-in father. It is thus also a polymorphism. The fourth variant was detected only in the proband (and, notably, was absent in his mother), when slow electrophoretic migration was used, conditions in which the $1 / 2$ polymorphism is barely detectable (fig. $2 a$ and $b$ ). On direct sequencing, we found an exonic single-base-pair change, A1244G. This results in a change of tyrosine 415 to a cysteine, in the predicted protein, called "myotubularin." This 